\title{
Regioselective Oxidative Ring Opening of Cyclopropyl Silyl Ethers: A Quantum Chemical Study
}

\author{
Heiko Rinderhagen, ${ }^{[a, c]}$ Jochen Mattay, ${ }^{*[a]}$ Rafael Nussbaum, ${ }^{[b]}$ and Thomas Bally ${ }^{*[b]}$
}

\begin{abstract}
In contrast to the structurally and configurationally stable alkyl- or aryl-substituted cyclopropyl radical cations, cyclopropyl silyl ethers undergo spontaneous ring opening upon oxidation whereby the endocyclic $\mathrm{C}-\mathrm{C}(\mathrm{O}$ TMS) bond is cleaved with remarkable selectivity. DFT calculations on 1trimethylsilyloxybicyclo[4.1.0]heptane
\end{abstract}

show that this selectivity arises from the topology of the potential surface of the corresponding radical cation which is initially generated in a very steep

Keywords: cations - cyclopropyl ethers $\cdot$ electron transfer $\cdot$ radicals . potential surfaces

\section{Introduction}

Cyclopropyl radical cations have attracted considerable interest over the last 30 years as intermediates for initiating radical or radical cationic cascade reactions as well as for mechanistic or structural reasons. ${ }^{[1-19]}$ Interestingly aliphatic or aromatic substituted cyclopropyl radical cations are-in absence of nucleophiles-structurally and configurationally stable. This has been demonstrated by CIDNP (chemically induced dynamic nuclear polarization) and by other transient spectroscopic experiments based on photoinduced electron transfer (PET).$^{[1,2,10]}$ The nucleophilic ring opening of cyclopropyl radical cations under PET conditions, which takes place under inversion of the carbon center which is attacked by the nucleophile (Scheme 1), has been studied mainly by the group of Dinnocenzo. ${ }^{[11-14]}$

[a] Dr. H. Rinderhagen, Prof. J. Mattay

Department of Chemistry, Bielefeld University

P.O. Box 100131, 33501 Bielefeld (Germany)

E-mail: oc1jm@uni-bielefeld.de

[b] R. Nussbaum, Prof. T. Bally

Department of Chemistry, University of Fribourg

Chemin du Musée 9, 1700 Fribourg (Switzerland)

E-mail: thomas.bally@unifr.ch

[c] Dr. H. Rinderhagen

Present Address: CILAG AG, Hochstrasse 201

8205 Schaffhausen (Switzerland) region of the potential surface from where the steepest descent leads to cleavage of the endocyclic rather than the lateral $\mathrm{C}-\mathrm{C}(\mathrm{OTMS})$ bond. Cleavage of the lateral bond leads to interesting conformational changes which are explored in detail. 


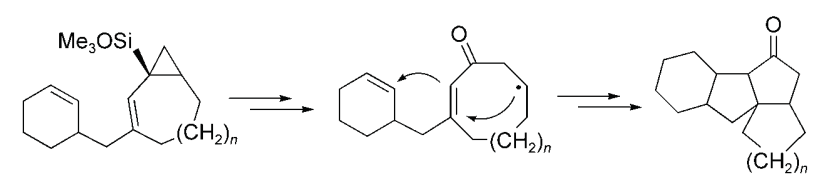

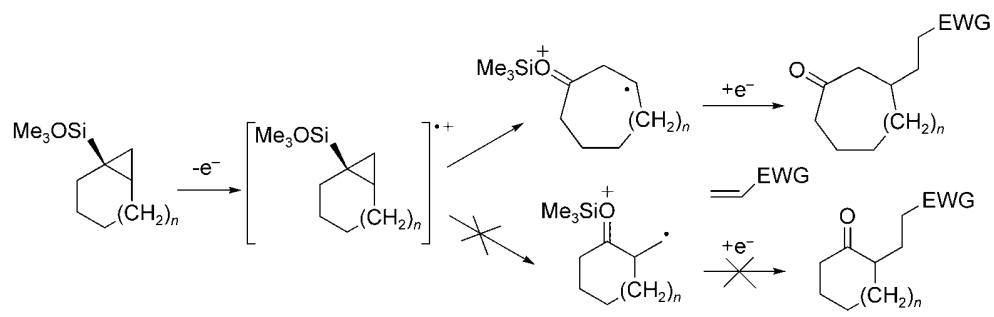

Scheme 2. Cyclopropyl silyl ether radical cations in preparative reactions. ${ }^{[1,18,19]}$ tential surface with one direction of negative curvature. This suggests the proximity of a saddle point and a corresponding transition state can indeed readily be found. In this structure $\mathrm{B}$ both $\mathrm{C}-\mathrm{C}$ bonds emanating from $\mathrm{C} 1$ are lengthened, although much less in the case of $\mathrm{C} 1-\mathrm{C} 6$ than $\mathrm{C} 1-\mathrm{C} 7$, while the $\mathrm{C}-\mathrm{O}$ bond is shortened to $1.31 \AA$. These changes are in accord with the nodal structure of the SOMO of B.

\section{Results and Discussion}

The HOMO of $\mathbf{1}$ is derived from the symmetric component of the degenerate Walsh MO of cyclopropane, the energy of which is raised in $\mathbf{1}$ through interaction with the $\mathrm{p}_{\pi}$ lone pair on the oxygen atom. It is bonding along $\mathrm{C} 1-\mathrm{C} 6$ as well as along $\mathrm{C} 1-\mathrm{C} 7$, and antibonding along $\mathrm{C} 6-\mathrm{C} 7$ and along $\mathrm{C} 1-$ O. Therefore we expect that ionization will lead to a lengthening of both the $\mathrm{C} 1-\mathrm{C} 6\left(r_{1}\right)$ and the $\mathrm{C} 1-\mathrm{C} 7$ bonds $\left(r_{2}\right)$, and a shortening of the $\mathrm{C} 6-\mathrm{C} 7$ and the $\mathrm{C} 1-\mathrm{O}$ bonds (Figure 1 ). It is, however, not possible to predict from the nodal structure of the MO from which an electron is removed on ionization whether any bond will break and, if yes, which of the two that are weakened.

Unconstrained geometry optimization of the vertically formed radical cation of $\mathbf{1}$ leads in fact to (partial) cleavage of the $\mathrm{C} 1-\mathrm{C} 6$ bond and results in structure $\mathrm{C}$ depicted in Figure 2, where $r_{1}$ has increased to $2.19 \AA$ and $r_{2}$ has slightly shrunk $\left(r_{2}=1.49 \AA\right)$. The singly occupied MO (SOMO) of $\mathrm{C}$ is now localized in the $\mathrm{C} 1-\mathrm{C} 6$ bond, that is, this retains the character of a one-electron bond, which is the reason why it is not completely broken. Note also that the $\mathrm{C}-\mathrm{O}$ bond length shortens from 1.41 to $1.28 \AA$ on ionization, in accord with the nodal structure of the SOMO.

If the $\mathrm{C} 1-\mathrm{C} 6$ bond is prevented from breaking during the relaxation of vertically ionized 1, for example, by constraining its length to never exceed $1.6 \AA$, this optimization leads to a (nonstationary) point that lies in a region of the poin $\AA$.

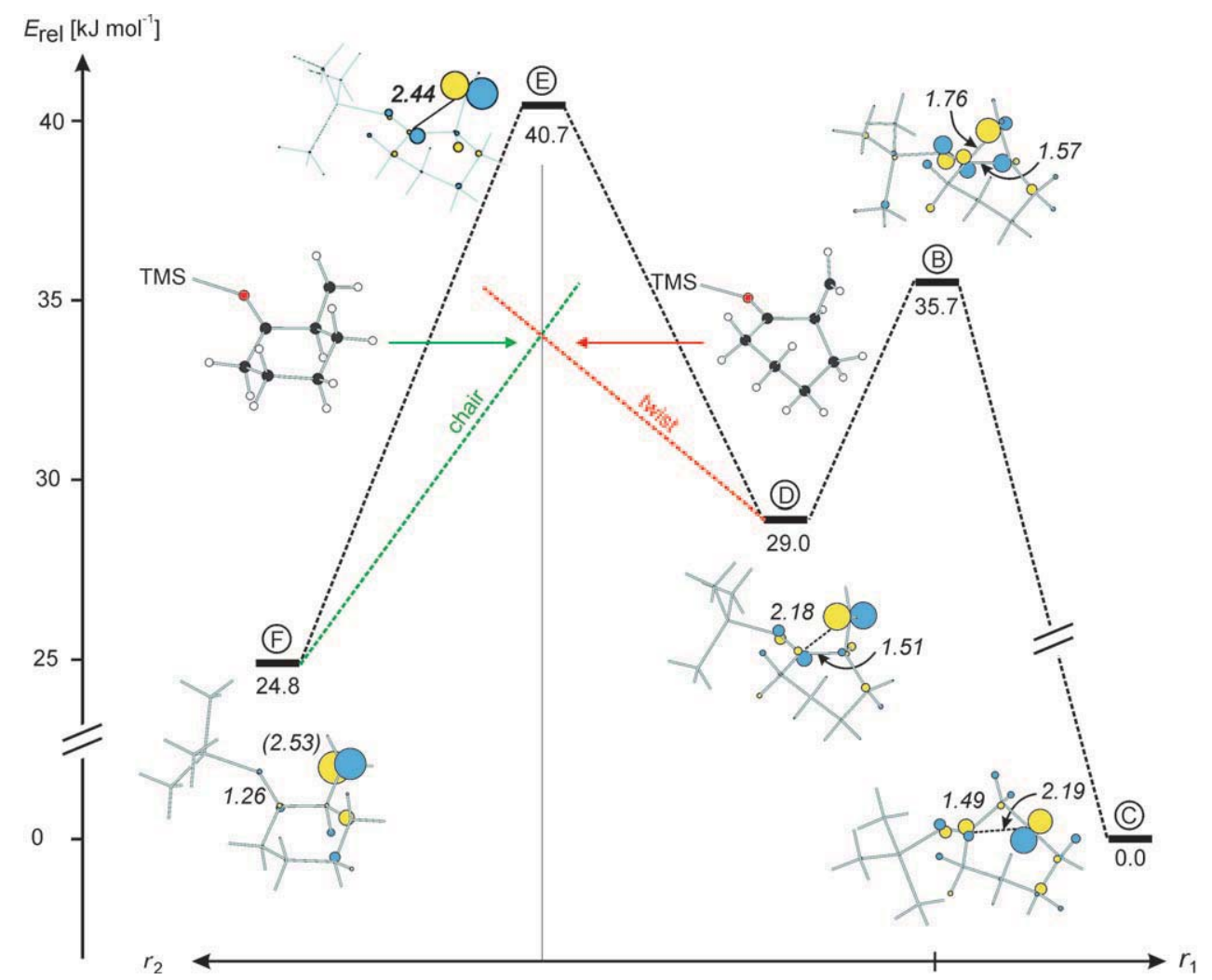

Figure 2. Stationary points on the potential surface of ionized $\mathbf{1}$ (cf., Figure 3). Numbers indicate bond lengths 
the value it had in neutral $\mathbf{1}\left(r_{1}=1.51 \AA\right)$, and the $\mathrm{C}-\mathrm{O}$ bond retains the partial double bond character $(1.28 \AA)$ it has already in structure B (see Figure 2).

In order to gain more detailed insight into the mechanism by which 1 decays on ionization, we decided to explore the potential surface of the radical cation in the space defined by coordinates $r_{1}$ and $r_{2}$ (see below). Thereby we found that, if $r_{2}$ is stretched beyond the equilibrium value of $2.18 \AA$ in structure $\mathrm{D}$, a new, more stable minimum $\mathrm{F}$ is eventually reached. In this structure the $\mathrm{C} 1-\mathrm{C} 7$ bond is completely broken, while the $\mathrm{C}-\mathrm{O}$ bond<smiles></smiles>
has attained the value it has in a protonated ketone (1.26 $\AA$ ). Formally structure $\mathrm{F}$ is a distonic radical cation, that is, a species where the spin and charge are localized on different atoms.

A Mulliken population analysis reveals indeed that in $\mathrm{F}$ $99 \%$ of the spin resides on the exocyclic $\mathrm{CH}_{2}$ moiety, whereas $68 \%$ of the positive charge is accommodated by the TMS group (the $\mathrm{O}$ atom effectively carries a negative charge of 0.59 which is, however, counterbalanced by a positive charge of similar magnitude on $\mathrm{C} 1$ ).

The passage from $\mathrm{D}$ to $\mathrm{F}$ is, however, not easily achieved because it necessitates a change from the twist conformation of the cyclohexane ring as it prevails in D to the chair structure of the same ring in F. On stretching of $r_{2}$ starting from $\mathrm{D}$ or compression of $r_{2}$ starting from $\mathrm{F}$ one finds that the surfaces of the two conformers cross along a seam that is indicated by a dot/dash line in Figure 3 and by the red and

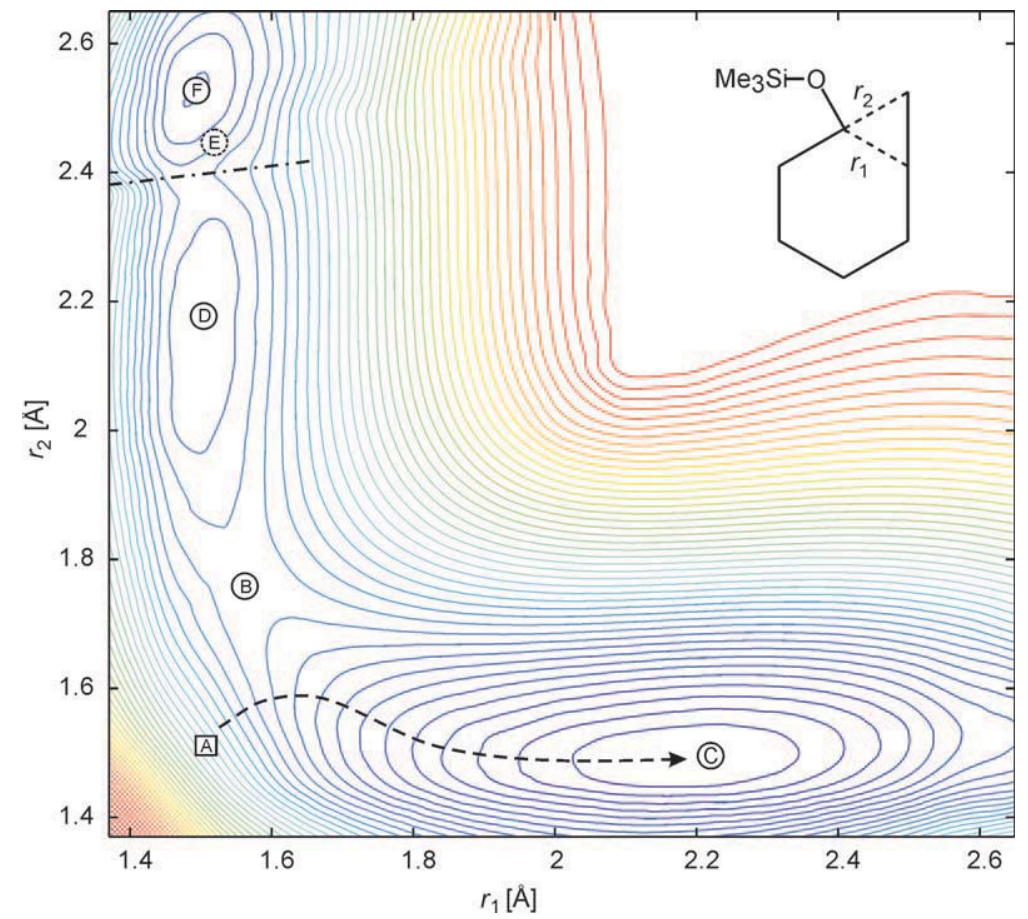

Figure 3. Three-dimensional potential surface for the decay of ionized 1, calculated by the B3LYP/6-31G* method. Contour lines are separated by $3 \mathrm{~kJ} \mathrm{~mol}^{-1}$. Point A corresponds to the equilibrium structure of neutral green dashed lines in Figure 2 (of course this crossing appears only as such in the two- or three-dimensional subspaces represented in the figures above).

Finding the transition state for the conformational change that gets the system from one to the other surface is not easy, but eventually we succeeded to locate a saddle point $\mathrm{E}$, about $6 \mathrm{~kJ} \mathrm{~mol}^{-1}$ above the lowest-energy point on the two surfaces for the values of $r_{1}$ and $r_{2}$ that prevail in E. Apart from the conformational change, which is largely responsible for the exothermicity of the rearrangement leading from $\mathrm{D}$ to $\mathrm{F}$ (in spite of the fact that the one-electron $\mathrm{C} 1-\mathrm{C} 7$ bond is cleaved), the only notable change is a lengthening of the $\mathrm{C} 1-\mathrm{C} 7$ bond by $0.26 \AA$ on the way from $\mathrm{D}$ to E.

In spite of this secondary rearrangement, the decay of ionized $\mathbf{1}$ to $\mathrm{C}$ is about $25 \mathrm{kcal} \mathrm{mol}^{-1}$ more exothermic than that to F, so one could say that the decay is driven thermodynamically. Inspection of the three-dimensional potential surface shown in Figure 3 shows, however, that the greater stability of $\mathrm{C}$ may not be the reason why this product is formed, instead of $\mathrm{D}$ or $\mathrm{F}$, but much rather the position of the vertically formed cation (structure A) relative to the transition state $\mathrm{B}$.

"Seen" from $\mathrm{D}$, this transition state is a very early one $\left(r_{2}\right.$ is still quite long while $r_{1}$ has hardly lengthened from the value it had in neutral 1), which follows of course from the large exothermicity of the $\mathrm{D} \rightarrow \mathrm{C}$ rearrangement. Consequently, rather than lying immediately below the vertically formed structure A, the transition state B is displaced towards $\mathrm{D}$ and will thus not be visited with any probability in the course of the decay of ionized $\mathbf{1}$, a process that will have a tendency to follow the path of steepest descent which leads directly into the potential valley directed towards $\mathrm{C}$ (see dashed line in Figure 3). Thus, the selectivity of the observed fragmentation has its origin in the topology of the potential surface depicted in Figure 3.

It is interesting to compare $\mathbf{1}$ to other substituted cyclopropanes. Dinnocenzo et al. have observed that on ionization 1phenyl-2-alkylcyclopropanes relax to structures where the $\mathrm{PhC}-\mathrm{C}$ bond is lengthened to $1.7-1.8 \AA$, but is never fully cleaved (in the absence of nucleophiles, aryl-alkyl cyclopropanes do not epimerize upon oxidation).$^{[14]}$ Obviously the OTMS group has a more profound influence on the electronic structure of the cyclopropane radical cation than the 
phenyl group, as cyclopropyl silyl ethers undergo a multitude of radical cationic fragmentation, addition and rearrangement reactions. ${ }^{[16-19]}$

Calculations on the model compound, 1-methoxy-1,2-dimethylcyclopropane $\mathbf{3}$, show that the strong distortions on ionization persist even if the OTMS group is replaced by an OMe group (but $\mathbf{3 a}$ is $6.3 \mathrm{kcalmol}^{-1}$ more stable than $\mathbf{3 b}$ ).
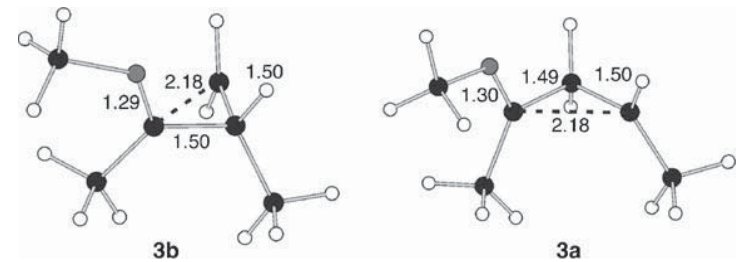

Actually, this distortive effect of the OMe group comes as a bit of a surprise: Due to its superior electron donor ability, one would expect this group to attenuate the effect of ionization of the cyclopropane structure, rather than to reinforce it. However, the partial separation of spin and charge which the breaking of the $\mathrm{OC}-\mathrm{C}$ bonds entrails leads to the formation of a very stable $\mathrm{C}=\mathrm{O}^{+} \mathrm{R}$ bond. This stability seems to overwhelm that provided by benzylic resonance in the case of a phenyl substituent, as can be seen from the below isodesmic reaction, which is exothermic by $13.5 \mathrm{kcal}$ $\mathrm{mol}^{-1}$.

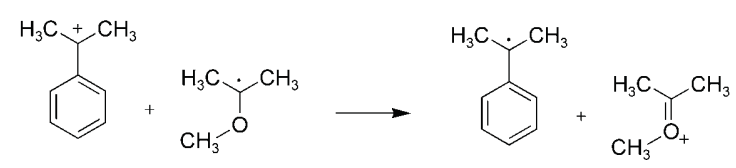

\section{Conclusion}

In contrast to alkyl- or aryl-substituted cyclopropanes, cyclopropyl silyl ethers undergo spontaneous $\mathrm{C}-\mathrm{C}$ bond cleavage on ionization, even in the absence of nucleophiles, which explains the chemistry of these compounds under oxidative conditions. Calculations on 1-trimethylsilyloxybicyclo[4.1.0]heptane 1, a bicyclic cyclopropyl silyl ether that was used in preparative studies, show that the choice of which of the two bonds cleaves depends on where the vertically formed radical cation is situated on the its potential surface. ${ }^{[22]}$

\section{Acknowledgements}

H.R. acknowledges the Studienstiftung des Deutschen Volkes for a predoctoral fellowship. Financial support was provided by the DFG and by the Swiss National Science Foundation (project No. 200020121747).
[1] H. D. Roth, M. L. M. Schilling, J. Am. Chem. Soc. 1980, 102, 7958 7960.

[2] H. D. Roth, M. L. M. Schilling, J. Am. Chem. Soc. 1981, 103, 72107217.

[3] H. D. Roth, M. L. M. Schilling, J. Am. Chem. Soc. 1983, 105, 68056808.

[4] H. D. Roth, M. L. M. Schilling, Can. J. Chem. 1983, 61, 1027-1035.

[5] H. D. Roth, T. Herbertz, P. S. Lakkaraju, G. Sluggett, N. J. Turro, J. Phys. Chem. A 1999, 103, 11350-11354.

[6] K. Krogh-Jespersen, H. D. Roth, J. Am. Chem. Soc. 1992, 114, 8388-8394.

[7] T. Herbertz, H. D. Roth, J. Am. Chem. Soc. 1998, 120, 11904-11911.

[8] P. C. Wong, D. R. Arnold, Tetrahedron Lett. 1979, 20, 2101-2104.

[9] C. E. Hudson, C. S. Giam, D. J. McAdoo, J. Org. Chem. 1993, 58, 2017-2019.

[10] S. B. Karki, J. P. Dinnocenzo, S. Farid, J. L. Goodman, I. R. Gould, T. A. Zona, J. Am. Chem. Soc. 1997, 119, 431-432.

[11] J. P. Dinnocenzo, D. R. Liebermann, T. R. Simpson, J. Am. Chem. Soc. 1993, 115, 366-367.

[12] J. P. Dinnocenzo, W. P. Todd, T. R. Simpson, I. R. Gould, J. Am. Chem. Soc. 1990, 112, 2462-2464.

[13] J. P. Dinnocenzo, T. R. Simpson, H. Zuilhof, W. P. Todd, T. Heinrich, J. Am. Chem. Soc. 1997, 119, 987-993.

[14] J. P. Dinnocenzo, H. Zuilhof, D. R. Liebermann, T. R. Simpson, M. W. McKechney, J. Am. Chem. Soc. 1997, 119, 994-1004.

[15] H. D. Roth in Electron Transfer in Chemistry Vol 2: Organic, Organometallic and Inorganic Molecules (Ed.: V. Balzani), Wiley-VCH, Weinheim 2001, pp. 55-132.

[16] H. Rinderhagen, J. Mattay, Chem. Eur. J. 2004, 10, 851-874.

[17] a) H. Rinderhagen, J. Mattay, J. Inf. Rec. Mater. 1998, 261-264; b) H. Rinderhagen, J. Grota, J. Mattay, J. Inf. Rec. Mater. 2000, 229233.

[18] H. Rinderhagen, P. A. Waske, J. Mattay, Tetrahedron 2006, 62, $6589-6593$.

[19] H. Rinderhagen, PhD Thesis, University of Bielefeld (Germany) 2002.

[20] All calculations were carried out using the B3LYP density functional method with the 6-31G* basis set, as implemented in the Gaussian 03 suite of programs. ${ }^{[21]}$ Due to the very flexible TMS group it was not always possible to completely converge geometry optimizations to the default thresholds with regard to the maximum displacement between cycles. Nevertheless, the nature of all stationary points was ascertained by second derivative calculations. Full Cartesian coordinates and energies, including thermal corrections, are available from the Supporting Information.

[21] Gaussian 03, Revision C.01, M. J. Frisch, et al., Gaussian, Inc., Wallingford CT, 2004; the full citation is given in the Supporting Information.

[22] A referee has pointed out that our gas-phase calculations may not be relevant to the experiments which were carried out mostly in acetonitrile. We have recalculated the energies of all stationary points by PCM-SCRF calculations in $\mathrm{CH}_{3} \mathrm{CN}$. The relative energies change indeed by $1-2 \mathrm{~kJ} \mathrm{~mol}^{-1}$ compared to those in Figure 2, but no qualitative change is noted. Reoptimization of the structure of $\mathrm{B}$ in $\mathrm{CH}_{3} \mathrm{CN}$ entailed only minor changes in the critical bond lengths. Details are available in the Supporting Information. 